\title{
LETTER
}

\section{Beta-lactam antibiotics in continuous infusion in critically ill patients}

\author{
Axel Jeurissen*1 and Robert Rutsaert ${ }^{2}$ \\ See related research by Taccone et al., http://ccforum.com/content/14/4/R126
}

We read with great interest Taccone and colleagues' article [1], published in a recent issue of Critical Care, on the insufficient $\beta$-lactam concentrations in the early phase of severe sepsis and septic shock. While we fully agree with the authors' findings, we would like to offer some remarks.

Only 18 of their 80 patients (22.5\%) were infected with Pseudomonas aeruginosa, but Taccone and colleagues used the European Committee on Antimicrobial Susceptibility Testing (EUCAST) minimal inhibitory concentration (MIC) breakpoints of $P$. aeruginosa to calculate the target pharmacokinetics (PK) profile in all of the patients. Because Enterobacteriaceae form a substantial part of infectious organisms in intensive care patients, it would be interesting to see how many patients would attain the PK profile for these microorganisms [2]. For cefepime, for instance, if the EUCAST sensitivity thres- hold of $1 \mathrm{mg} / \mathrm{L}$ were used, 17 of 19 patients (89\%) would attain the target PK profile as compared with 3 of 19 patients (16\%) for $P$. aeruginosa. Of course, we agree that, in an empirically started antibiotic regimen, the organisms, let alone the MIC, are not known to the clinician.

Furthermore, the data of Taccone and colleagues should be interpreted in light of local epidemiology and resistance data. In a Belgian multicenter study, all $P$. aeruginosa strains isolated from patients hospitalized in the intensive care unit (ICU) had an $\mathrm{MIC}_{90}$ (MIC required to inhibit the growth of $90 \%$ of organisms) for meropenem of $0.12 \mathrm{mg} / \mathrm{L}$ [3]. With this MIC, even more than $75 \%$ of the patients would have attained the target PK profile. In addition, we think that the initial loading dose should be followed immediately by an extended or continuous infusion in order to obtain an optimal PK/ pharmacodynamics (PK/PD) profile [4].

\section{Authors' response}

Fabio Silvio Taccone, Jean-Louis Vincent and Frédérique Jacobs

We thank Jeurissen and Rutsaert for their interest in our study [1] and would like to reply to the important points they raise. In our patient population, one third of documented infections were due to $P$. aeruginosa as microbiological samples remained negative in $30 \%$ of patients with sepsis. Indeed, $P$. aeruginosa is frequently isolated in patients with comorbid illnesses or indwelling catheters or who are on mechanical ventilation or undergoing surgery, all of these conditions being typical in ICU patients [5]. Pseudomonas infections are associated with the highest mortality rate in this ICU patient population. For all of these reasons, it seems logical to develop an

\footnotetext{
*Correspondence: axel.jeurissen@gza.be

'Department of Medical Microbiology, GZA St. Vincentius, St. Vincentiusstraat 20, 2018 Antwerp, Belgium

Full list of author information is available at the end of the article
}

empirical strategy that targets this pathogen in patients with nosocomial infections.

We agree that in vitro studies on Pseudomonas susceptibility may show MICs that are much lower than the upper threshold of sensibility proposed by the EUCAST for carbapenems. However, in all epidemiologic studies, only the first isolated strain of P. aeruginosa is considered for MIC determination. Besides having an intrinsic resistance to a wide range of antimicrobials, Pseudomonas is able to acquire resistance via several mechanisms or under antimicrobial pressure. A recent study showed that Pseudomonas strains isolated from ICU patients are able to progressively increase the in vitro MIC level to different antibiotics during therapy [6].

Finally, we agree that the extended or continuous infusion of $\beta$-lactams can optimize the PK/PD profile of these drugs. Unfortunately, as only retrospective studies have provided evidence in favor of continuous infusion over intermittent infusion (especially in pathogens with higher 
MICs and in ventilator-associated pneumonia [7]), a prospective study in this setting is warranted.

\section{Abbreviations}

EUCAST, European Committee on Antimicrobial Susceptibility Testing:

$I C U$, intensive care unit; MIC, minimal inhibitory concentration; PD,

pharmacodynamics; PK, pharmacokinetics.

\section{Competing interests}

FST, FJ, and J-LV have received lecture honoraria from AstraZeneca (London,

UK). J-LV is on the speakers' list of GlaxoSmithKline (Uxbridge, Middlesex, UK).

The other authors declare that they have no competing interests.

\section{Author details}

'Department of Medical Microbiology, GZA St. Vincentius, St. Vincentiusstraat 20, 2018 Antwerp, Belgium. ${ }^{2}$ Department of Intensive Care Medicine, GZA St. Vincentius, St. Vincentiusstraat 20, 2018 Antwerp, Belgium.

Published: 26 October 2010

\section{References}

1. Taccone FS, Laterre PF, Dugernier T, Spapen H, Delattre I, Witebolle X, De Backer D, Layeux B, Wallemacq P, Vincent JL, Jacobs F: Insufficient $\beta$-lactam concentrations in the early phase of severe sepsis and septic shock. Crit Care 2010, 14:R126.

2. Vincent JL, Rello J, Marshal J, Eliezer S, Anzueto A, Martin CD, Moreno R, Lipman J, Gomersall C, Sakr Y, Reinhart K for the EPIC II Group of Investigators:
International study of the prevalence and outcomes of infection in intensive care units. JAMA 2009, 302:2323-2329.

3. Belgian Isepamycin Multicentre Group: Comparative in vitro activity of isepamycin and other antibiotics against gram-negative bacilli from intensive care units (ICU) in Belgium. Acta Clin Belg 2001, 56:307-315.

4. Roberts JA, Lipman J, Blot S, Rello J: Better outcomes through continuous infusion of time-dependent antibiotics to critically ill patients? Curr Opin Crit Care 2008, 14:390-396.

5. Page MG, Heim J: Prospects for the next anti-Pseudomonas drug. Curr Opin Pharmacol 2009, 9:558-565.

6. Riou M, Carbonnelle S, Avrain L, Mesaros N, Pirnay JP, Bilocg F, De Vos D, Simon A, Piérard D, Jacobs F, Dediste A, Tulkens P, Van Bambeke F, Glupczynski $Y$ : In vivo development of antimicrobial resistance in Pseudomonas aeruginosa strains isolated from the lower respiratory tract of intensive care unit patients with nosocomial pneumonia and receiving antipseudomonal therapy. Int J Antimicrob Agents 2010 Oct 4. [Epub ahead of print].

7. Lorente L, Jiménez A, Palmero S, Jiménez JJ, Iribarren JL, Santana M, Martin MM, Mora ML: Comparison of clinical cure rates in adults with ventilatorassociated pneumonia treated with intravenous ceftazidime administrated by continuous or intermittent infusion: a retrospective, nonrandomized, open-label, historical chart review. Clin Ther 2007. 29:2433-2439.

doi:10.1186/cc9288

Cite this article as: Jeurissen A, Rutsaert R: Beta-lactam antibiotics in continuous infusion in critically ill patients. Critical Care 2010, 14:446. 